\title{
Género y ficción televisiva: ¿Nuevas feminidades y masculinidades en la serie mexicana Club de Cuervos?
}

\section{Genre and television fiction: New femininities and masculinities in the Mexican series Club de Cuervos?}

\section{Gênero e ficção televisiva: ¿Novas feminilidades e masculinidades na série mexicana Club de Cuervos?}

Karla Contreras Tinoco, Universidad de Guadalajara, Jalisco, México (karla.ctinoco@academicos.udg.mx)

Laura Alfaro Beracoechea, Universidad de Guadalajara, Jalisco, México (nadhielii.alfaro@academicos.udg.mx)

RESUMEN | El objetivo de este estudio es analizar las características identitarias, roles, ámbitos de actuación y estereotipos de género en la serie de ficción televisiva mexicana Club de Cuervos (2015), proyectada en un servicio de streaming. De manera independiente, dos jueces realizaron un análisis de su contenido, basado en un modelo representacional temático de la primera temporada. En los resultados destaca que los personajes hombres se presentan como consejeros, exitosos, propositivos, abnegados e hipersexualizados. Asimismo, tienen mayor presencia en el espacio público y establecen más homosocialidad. Las mujeres, en cambio, se presentan como amorosas, tiernas, hipersexualizadas, aparecen más en el espacio privado y tienen más relaciones heterosociales. Se discuten las implicancias de las diferencias entre género en las varias categorías y de las nuevas masculinidades en la representación de los hombres mexicanos. Se concluye que la serie intenta romper con las masculinidades y feminidades hegemónicas en características identitarias, presentando nuevos modelos de masculinidad en distintos personajes. También reproduce roles y figuras de una identidad de género constituida por el ser para otros y subordinada a los hombres.

PALABRAS CLAVE: identidad de género; roles; ficción televisiva; análisis de contenido; servicios de streaming. 
ABSTRACT / This study aimed to analyze the identity characteristics, roles, fields of action, and gender stereotypes in a television fiction series broadcasted on a streaming service. Two judges independently performed a content analysis based on a thematic representational model from the first season of the Mexican series Club de Cuervos. The results show that the male characters are presented as advisers, accomplished, purposeful, self-sacrificing, and hypersexualized. In addition, they have a higher presence in public space and establish more homosociality. On the other hand, women are presented as loving, tenders, hypersexualized, appear more in private sites and have more heterosocial relationships. We discuss the implications of gender differences in the various categories and new masculinities in the representation of Mexican men. It stands out, in the conclusions, that the series tries to set itself apart from hegemonic masculinities and feminities in identity characteristics, presenting new models of masculinity in different characters. It also reproduces roles and figures of a gender identity constituted by being for others and subordinate to men.

KEYWORDS: gender identity; roles; fictional television; content analysis; streaming services.

RESUMO | O intuito deste estudo é analisar as características de identidade, papéis, âmbitos de atuação e estereótipos de gênero na série de ficção televisiva mexicana Club de Cuervos (2015), exibida em um serviço de streaming. De maneira independente, dois juízes conduziram uma análise de conteúdo, baseada em um modelo de representação temática da primeira temporada. Os resultados mostram que as personagens masculinas são apresentadas como conselheiros, bem-sucedidos, proativos, abnegados e hipersexualizados. Eles também têm uma presença maior no espaço público e estabelecem mais homossocialidade. As mulheres, por outro lado, são apresentadas como amorosas, ternas, hipersexualizadas, aparecem mais no espaço privado e têm relações mais heterossexuais. As implicações das diferenças de gênero nas diversas categorias e das novas masculinidades na representação dos homens mexicanos são discutidas. Conclui-se que a série tenta romper com as masculinidades e feminilidades hegemônicas em características de identidade, apresentando novos modelos de masculinidade em diferentes personagens. Além disso, reproduz papéis e figuras de uma identidade de gênero constituída por ser para os outros e subordinada aos homens.

PALAVRAS-CHAVE: identidade de gênero; papéis; ficção televisiva; análise de conteúdo; serviços de streaming. 


\section{INTRODUCCIÓN}

En el mundo opera un orden social de género que establece un marco organizador simbólico de jerarquías, normas, roles, atribuciones, características y posibilidades de experiencia diferenciados para los sujetos según su sexo. Este orden social de género interviene en un espacio y tiempo determinado, está basado en la diferencia sexual, no es natural -aunque muchas veces está naturalizado (Palomar, 2007)y es problemático porque legitima y reproduce desigualdades y diferencias entre hombres y mujeres, además de estar sustentado en relaciones de poder (Scott, 1999, 2008). A su vez, está estrechamente vinculado con la producción de las identidades de género. En torno al ser mujer, socialmente se han construido e impuesto ciertas características y cualidades, tales como el gusto y la habilidad de cuidado de los otros, la capacidad, deseo e instinto maternal, la sensibilidad y expresividad de emociones, y la relegación al espacio privado (Castañeda, 2016; Ortega-Lorenzo \& Simelio, 2012). En cambio, a los hombres se les ha asociado con el espacio público y se les han atribuido cualidades como fortaleza, racionalidad, capacidad competitiva, el ser para sí, la proveeduría económica, y la dificultad para demostrar emociones, entre otros (Ramírez, 2019).

Estas características identitarias de género han decantado en roles específicos esperados y asociados a las mujeres y a los hombres. Cabe señalar que por roles sociales entendemos las tareas, obligaciones, responsabilidades o actividades que son realizadas por los miembros de un grupo, que pueden haber sido asignadas formalmente, impuestas o adquiridas (Baron \& Byrne, 2005). En los casos en los que los roles han sido impuestos, puede haber conflicto o inconformidad porque las personas no se sienten identificadas o conformes con el rol asignado o porque no les gusta. A veces, dichos roles generan jerarquías o estratificaciones y ofrecen prestigio; en otras ocasiones, conllevan estigmas sociales. Las personas a las que se les ha adjudicado un rol que no es compatible con sus intereses, habilidades o gustos pueden experimentar malestar, inconformidad y molestia (Baron \& Byrne, 2005).

En estrecha relación con los roles de género están los estereotipos de género, que adjudican características, cualidades o rasgos distintivos a las personas por su sola adscripción a un grupo social (Park et al., 1991). Los estereotipos son problemáticos, porque ofrecen una imagen homogénea y simplista de un grupo (por ejemplo, de los hombres). Es incluso común que si las personas muestran rasgos que no son consistentes con los estereotipos que la sociedad les ha adjudicado, estos sean rechazados o modificados sutilmente para que su comportamiento se adecue al estereotipo. Asimismo, los estereotipos influyen en los juicios, evaluaciones y expectativas sobre los otros (Baron \& Byrne, 2005). 
Es frecuente la presencia y existencia de estereotipos de género que ofrecen rasgos positivos y negativos para ambos sexos. Los estereotipos, además, se vinculan con prejuicios (sutiles y explícitos) y con comportamientos violentos. Por ejemplo, el sexismo hostil, mediante el cual se les adjudican características negativas a las mujeres (y se les considera menos valiosas que los hombres), o bien el sexismo benevolente, que consiste en pensar que las mujeres merecen protección, son superiores a los hombres en diversos aspectos (superioridad moral, pureza, recato, tienen mejor gusto, entre otros) y son importantes y necesarias para que los hombres alcancen la felicidad (Baron \& Byrne, 2005).

\section{Series de televisión y género}

Durante años, las relaciones cara a cara se consideraron fundamentales para construir la realidad social (Berger \& Luckman, 1968; Goffman, 2001); se pensaba que, por medio de esas interacciones, los sujetos construían su identidad, recibían y otorgaban a otros una posición social, se les adscribía a una categoría social, asumían roles, se les transmitían (y reproducían) normas y modos de vida deseables. Ahora, muchas de las interacciones ocurren mediadas por los medios de comunicación (televisión, cine, publicidad, música, etc.) y las redes sociales digitales (Caballero-Gálvez \& Herrero-Jiménez, 2017).

Desde las últimas décadas del siglo XX, en particular, los medios de comunicación irrumpieron en la vida de forma masiva y marcada, lo cual generó que se modificaran los modos de comunicación interpersonal, las relaciones sexoafectivas (Caballero-Gálvez \& Herrero-Jiménez, 2017) y la subjetividad, mediante la producción de deseos, modelos, recursos, aspiraciones, formas de vida, estilos de vestimenta y de relación (Bueno-Fischer, 2006; Richard, 2006). Al acceder a los medios de comunicación (radio, televisión, servicios de streaming, etc.), las personas integran en sus esquemas cognitivos ideas, estereotipos y roles que se convierten en modelos para construir su identidad y orientar sus interacciones sociales (Galán, 2006; Guarinos et al., 2019; Menéndez \& Zurian, 2014; Simelio, 2010).

Diversos autores (Galán, 2006; Bueno-Fischer, 2007; Pacheco, citado en HidalgoMarí, 2017) sostienen que los productos de los medios de comunicación también son resultado y reflejo de la sociedad, pues los guionistas y los escritores extraen de la vida cotidiana sus historias, argumentos y temáticas. Por ello se dice que la relación de influencia entre sociedad y medios de comunicación es bidireccional y mutuamente construida y reactualizada, lo cual muestra el doble rol y relevancia que adquieren los medios de comunicación en la actualidad. Aun cuando los contenidos audiovisuales parten de argumentos reales y cercanos a la vida cotidiana, muchas veces se recurre a la exageración y a los estereotipos para contar las historias y mostrar perfiles deseables de ser profesional o deportista, por ejemplo (Galán, 2006). 
Para Guarinos y sus colegas (2019), los medios de comunicación inciden en la manera en la que los individuos construyen su identidad de género, ya que mediante ellos descubren, reconocen, apropian y reproducen qué es lo femenino y qué es lo masculino. Diversos estudios (Ortega-Lorenzo \& Simelio, 2012; MateosPérez \& Ochoa, 2021) han demostrado que en las series de televisión se reproducen estos roles y estereotipos de género. Desde los años 80 han proliferado los estudios científicos que analizan el tema de la construcción y presentación de género en los medios de comunicación (Hidalgo-Marí, 2017). Sin embargo, para Fonseca (2017) este interés viene de los años 70, con lo que se conoce como teoría fílmica feminista. La pionera de este grupo de estudios fue Claire Johnston, quien realizó una crítica semiótica sobre el rol del cine clásico en la construcción ideológica de la mujer.

Los primeros trabajos acerca de roles y estereotipos de género en la ficción televisiva se concentraron en países anglosajones así que, según Galán (2007), el interés en los países de habla hispana es relativamente reciente (desde los años 90). Incluso, en habla hispana no es hasta finales de los 90 que surgieron las series profesionales, en las que se comenzó a mostrar a mujeres en puestos profesionales de responsabilidad que tradicionalmente eran ocupados por hombres, por ejemplo, mujeres policías, detectives, doctoras, abogadas, etc. (Galán, 2007).

En la actualidad, los estudios de representación social de las mujeres y de los hombres en medios de comunicación son frecuentes (por ejemplo, Bonavitta \& de Garay, 2019; Caballero-Gálvez \& Herrero-Jiménez, 2017; Hidalgo-Marí, 2017; Galán, 2006; Fonseca, 2017; Menéndez \& Zurian, 2014; Pérez, 2017, Mateos-Pérez \& Ochoa, 2016, 2021; Ortega-Lorenzo \& Simelio, 2012; Simelio, 2010). En la mayoría de estos trabajos se sostiene que en los medios de comunicación se muestran y reproducen contenidos androcéntricos y patriarcales.

Asimismo, a partir de una profunda y exhaustiva revisión de trabajos acerca de ficción televisada, Menéndez y Zurian (2014) señalan que es común encontrar que las mujeres aparecen menos número de veces que los hombres, así como la presentación de estereotipos de género sin una perspectiva crítica. Por ejemplo, a las mujeres se les representa desde figuras y roles pasivos y como objetos de deseo o atención de otros, mientras que a los hombres se les presenta como poderosos y activos (Bonavitta \& de Garay, 2019; Fonseca, 2017). También es frecuente ver que las mujeres ocupen roles con posiciones subordinadas o bien arquetipos y estereotipos que las dibujan desde aspectos negativos (Pérez, 2017), escenas y tramas que las cosifican, las sexualizan y exaltan la belleza o los valores de abnegación (Menéndez \& Zurian, 2014).

A su vez, los estudios sobre representaciones de género sostienen varias cosas: primero, que no es habitual que se destaque o remarque el rol de esposa de las mujeres, pero sí el de madres (Sánchez et al., citado en Hidalgo-Marí, 2017). Segundo, que 
las tramas que envuelven a las mujeres maduras están relacionadas con la familia tradicional y el amor romántico (Hidalgo-Marí, 2017). Tercero, que en las ficciones que presentan a mujeres trabajadoras, estas desarrollan y desempeñan su rol doméstico primordialmente, están masculinizadas o bien son súper mujeres que combinan actividades y obligaciones tanto laborales como familiares (Hidalgo-Marí, 2017). Es frecuente que ocupen roles y tareas subordinadas a hombres, con roles de directores y responsables de empresas (Ortega-Lorenzo \& Simelio, 2012). Un tópico recurrente es el de mujeres técnicas, que desempeñan su labor con eficacia y eficiencia pero que se encuentran bajo el mando de un hombre poderoso o carente de habilidades (Hidalgo-Marí, 2017). En cuarto lugar, las mujeres se muestran como emocionales, pasivas, maternales, en espacios privados, con una sexualidad para otros, mientras que los hombres se presentan rodeados de atributos de raciocinio, liderazgo y acción en los espacios públicos (Bonavitta \& de Garay, 2019; Galán, 2007; Hidalgo-Marí, 2017; Menéndez \& Zurian, 2014). En los casos en los que se presentan mujeres fuertes, habitualmente es desde su categoría de madres donde encuentran coraje o fortaleza para hacer frente a los problemas familiares. Sin embargo, se las muestran frustradas y cansadas por el cuidado de los hijos; incluso, se presentan como dependientes emocionales de los hombres (Bonavitta \& de Garay, 2019; Hidalgo-Marí, 2017).

Con respecto a los roles que desempeñan los hombres en las series de televisión, han sido personajes masculinos con ciertos matices a los ejes hegemónicos con los que se reconoce a los hombres (Mateos-Pérez \& Ochoa, 2021). Son obedientes y tolerantes con sus esposas. Sin embargo, terminan retomando el control de la relación, encontrando la fortaleza que todo hombre debe tener y situando a las mujeres en su lugar, es decir, la casa (Menéndez \& Zurian, 2014).

Por las razones anteriores, según Galán (2006), sigue siendo necesario analizar la influencia de los medios de comunicación en temáticas como las diferencias de roles sexuales y estereotipos de género en las representaciones de hombres y mujeres en la televisión.

Para Menéndez y Zurian (2014), el hecho de que se coloque a las mujeres como protagonistas de las series y programas televisivos ya es en sí mismo una expresión de transgresión a su invisibilización en los medios. Los autores sostienen que la ficción televisiva contemporánea, aun cuando no ha roto del todo los estereotipos de género, sí ha incluido nuevos discursos ficcionales y está mostrando nuevos roles y tipos de ser hombre y mujer. Lotz (2006, citada en Menéndez y Zurian), sostiene que desde los años 90 ha habido cambios y transiciones en las imágenes comunes y habituales que se socializan de las mujeres en las películas y teleseries, al presentar una variedad más amplia de posibilidades de vidas, papeles más independientes y, en general, a visibilizar más las características positivas de las mujeres. 
Asimismo, si bien se han documentado ampliamente las diferencias de género que se muestran y difunden en series de televisión y películas, actualmente es más común el uso de Internet que el de la televisión, sobre todo en la población más joven (Suárez-Cousillas et al., 2019). En México, mientras que el tiempo diario para ver televisión decreció en los últimos 10 años, el consumo de contenido a través de Internet ha aumentado gradualmente (Ortiz, 2020). Finalmente, consideramos oportuno revisar en el marco de las características identitarias de género las relaciones de homosociabilidad y heterosociabilidad de los hombres y las mujeres en la ficción televisiva, ya que las consideramos como un indicador de las relaciones asimétricas entre géneros. De este modo, pueden ayudar a comprender las construcciones hegemónicas de masculinidad y de feminidad. La homosociabilidad se refiere a las atracciones o interacciones no sexuales entre hombres o mujeres por miembros de su mismo sexo, mientras que la heterosociabilidad se orienta a las atracciones no sexuales con miembros del sexo opuesto (Bird, 1996). Adicionalmente, un indicador de homosociabilidad para las mujeres es el Test de Bechdel-Wallace, que consiste en identificar: 1) que haya al menos dos personajes mujeres; 2) que ambas establezcan una conversación entre sí y sin un hombre presente, y 3) que el tema de conversación no sea acerca de un hombre (Freitas et al., 2016).

\section{Servicios de streaming y contextualización del estudio}

Internet ha modificado el acceso y la relación de los consumidores con las series televisivas o en plataformas de streaming, ya que la audiencia participa en blogs, foros, chats, redes sociales -tanto en páginas oficiales como no oficiales-. Incluso, los fans han escrito guiones alternativos basados en la historia, los personajes y temáticas de una serie, y los cuelgan en plataformas como YouTube. El éxito y revolución que ha tenido este tipo de productos audiovisuales creados específicamente por Internet ha generado el aumento y la diversidad de los servicios y plataformas que las ofrecen (Netflix, HBO, Blim, entre otras). Estas ofrecen la posibilidad de conectarse en dispositivos móviles, no almacenan u ocupan espacio del ordenador, hay un acceso casi ilimitado al contenido, sin estar sujeto a horarios de programación (Siri, 2016; Suárez-Cousillas et al., 2019). Asimismo, son plataformas que tienen como público objetivo principalmente a los jóvenes o los adultos jóvenes (Simelio, 2010).

Netflix es el principal servicio de entretenimiento audiovisual de pago accesible vía Internet, tiene presencia en 190 países y, aproximadamente, 130 millones de suscriptores. Algunas de las razones de su popularidad son que es un servicio relativamente económico y que usa algoritmos y estrategias de sugerencias de interés a los usuarios de series, películas o documentales, además de ofrecer la posibilidad de hacer binge-watching (Siri, 2016; Suárez-Cousillas et al., 2019). 
La primera serie de habla hispana producida por la plataforma de SVOD Netflix fue Club de Cuervos (Chiver et al., 2015-2019; Nájar, 2015). Comenzó a proyectarse en agosto de 2015 y cuenta con cuatro temporadas que relatan las tensiones y disputas entre los hermanos Isabel y Chava por la presidencia de un equipo de fútbol. Chava es un joven que gusta de las fiestas, consume sustancias adictivas, es soñador y hasta antes de la muerte de su padre había estado poco implicado en lo concerniente a administrar el equipo. En cambio, Isabel, la hija mayor, ha tenido una participación activa e implicada en todo lo referente a la conducción del equipo. Además, goza de la confianza de su padre porque es responsable. La historia inicia cuando el padre de estos personajes muere a causa de un infarto provocado por un disgusto con Chava. Pese a ello, tanto familiares como otros miembros de la mesa directa del equipo asumen sin el menor cuestionamiento que Chava será el presidente del equipo. A lo largo de la serie, Isabel intenta demostrar que ella es la verdadera merecedora de la presidencia. Si bien la descripción que ofrece Netflix para la serie en su página web se centra en la lucha de poder y la rivalidad entre hermanos (Netflix, n.d.), es claro que presentar una mujer coprotagonista en la serie cuya premisa implica que es ella la mejor preparada para un puesto en un contexto primordialmente masculino da pie al análisis de las implicancias del género y sus características e interrelaciones. Analizamos esta serie porque es una de las primeras series mexicanas protagonizadas por una mujer, y que muestra a una mujer que lucha por estar y ocupar un puesto directivo en una empresa.

\section{MÉTODO}

En este trabajo, el objetivo fue analizar las diferencias en las características identitarias, roles, ámbitos de actuación y estereotipos de género, así como las relaciones de homosociabilidad y heterosociabilidad en hombres y mujeres en el programa de ficción televisiva Club de Cuervos.

En este estudio, planteamos de forma cuantitativa las diferencias por sexo en las distintas categorías de análisis, una aproximación novedosa considerando la revisión de la literatura existente sobre las representaciones de género, que han sido mayormente desde un enfoque cualitativo (Bonavitta \& de Garay, 2019) o incorporando solamente frecuencias (Galán, 2007).

Se analizó la primera temporada de la serie, constituida de 13 episodios. Se realizó un análisis de contenido basado en un modelo representacional temático, que consiste en relevar cuantitativamente el contenido lexical del discurso, y ver la cantidad y sentido de las palabras en cuanto a un núcleo de sentido. Para ello, se hizo un conteo de frecuencia de las unidades de significación, con el que 
se observa y evalúa la mayor o menor presencia de determinados temas, lo que muestra la relevancia y valor de referencia que se le otorga a ciertos discursos e imágenes de sentido (Souza, 2009).

Siguiendo a Souza (2009), el análisis siguió tres etapas:

1. Preanálisis: se determinaron las unidades de registro (palabras, conductas observables, frases), los contextos y los lugares de actuación. Además, se establecieron las formas de categorización, codificación y se definieron los conceptos teóricos más generales que orientan los análisis.

2. Etapa de exploración del material: se buscaron expresiones, palabras significativas en los episodios y se codificaron en las matrices de observación.

3. Tratamiento de los resultados e interpretación: se hicieron operaciones simples (porcentajes, frecuencias) y estadística correlacional, como base para las interpretaciones.

Se elaboró una matriz de observables, constituida por seis ejes: 1) datos sociodemográficos, 2) características identitarias de género, 3) roles, 4) hipersexualización del personaje, 5) relación entre personajes, y 6) reproducción de tópicos. Las categorías de cada eje eran de opción múltiple. Asimismo, se elaboró una lista de operacionalización de cada categoría y un documento descriptor de los ejes (tabla 1).

Cada episodio fue observado por dos revisores de manera independiente. El llenado de la matriz se hizo simultáneo a la revisión del episodio, así que en cada escena se fue poniendo en pausa el episodio y procediendo a la captura de la información.

Posteriormente, los datos fueron capturados en Excel y en el programa SPSS v. 21. Una vez obtenido el material, se realizaron varias pruebas estadísticas descriptivas, análisis de frecuencias y diferencias de medias por sexo. En el caso de la categoría de reproducción de tópicos, realizamos una descripción de cada uno de los personajes que identificamos que encarnaron un estereotipo, ya que notamos que, por un lado, una diferencia de medias no nos permitiría retratar la complejidad del tópico representado y, por otro, nos interesaba precisar las cualidades y características con las que se presentó ese estereotipo. La fiabilidad interjueces para las categorías se probó con el estadístico alpha de Krippendorff (2004), obteniendo un nivel de acuerdo mayor al mínimo aceptable por los autores $(<=, 81)$ utilizando el macro KALPHA para SPSS (Hayes \& Krippendorff, 2007). Adicionalmente, se aplicó el test de Bechdel-Wallance en cada capítulo. 
Categoría

\begin{tabular}{|c|c|}
\hline \multirow{6}{*}{$\begin{array}{l}\text { Datos } \\
\text { sociodemográficos }\end{array}$} & $\begin{array}{l}\text { Sexo (1= Hombre; } 2=\text { Mujer; } 3=\text { No binario }) \\
\text { Grupo de edad (1= Niño } 0 \text { a } 12 \text { años; } 2=\text { Adolescente } 13 \text { a } 17 \text { años; } 3=\text { Joven }\end{array}$ \\
\hline & Color de piel ( $1=$ Moreno $2=$ Blanco; $3=$ Afrodescendiente $)$ \\
\hline & Ocupación laboral (1= Servicios; $2=$ Profesional; $3=$ Empresario; \\
\hline & del hogar; $5=$ Deportes; $6=$ Periodismo/comunicación; \\
\hline & $7=$ Gobierno; $8=$ \\
\hline & $\begin{array}{l}\text { Orientación sexual }(1=\text { Heterosexual; } 2=\text { Indefinido; } 3=\text { Lesbiana; } 4=\text { Bisexual; } \\
\quad 5=\text { Homosexual; } 6=\text { Transgénero; } 7=\text { Transexual; } 8 \text { = Pansexual) }\end{array}$ \\
\hline
\end{tabular}

\section{Código y operacionalización (OP)}

OP: $0=$ No aparece; $1=$ Sí aparece

Exitoso(a)
Trabajador(a)
Amoroso(a)
Tierno(a)
Extrovertido(a)
Servicial
Abnegado(a)
Agresivo(a)
Paciente

Fuerte Inteligente Detallista Generoso(a) Propositivo(a) Introvertido(a) Solidario(a) Resolutivo(a) Soñador(a)

\begin{tabular}{cc}
\hline OP: $0=$ No aparece; $1=$ Sí aparece \\
Toma de decisiones \\
Proveedor(a) \\
Consejero(a) \\
Cuidador(a) \\
Parásito
\end{tabular}

Tabla 1. Listado de códigos para categorizar las variables

Fuente: Elaboración propia. 


\section{RESULTADOS}

En la serie se registraron 74 personajes, de los cuales $53(71,6 \%)$ fueron hombres y $21(28,4 \%)$, mujeres. Los personajes fueron contabilizados siempre y cuando, además de aparecer en la escena, mantuvieran un diálogo. Este criterio de inclusión resultó útil para caracterizar a los personajes; sin embargo, es relevante mencionar que se dejó fuera del análisis a personajes de fondo. Resulta llamativo que estos personajes sin diálogo en su mayoría son mujeres: en unos casos son asistentes del hogar que llevan a cabo tareas domésticas y, en muchos otros, presentan características de hipersexualización y aparecen acompañando a los deportistas en fiestas, riendo, desnudas o semidesnudas. También se identificaron jugadores del equipo de fútbol que no tenían conversación con otros personajes, particularmente hombres jóvenes.

En la tabla 2 se muestra la información acerca de la categoría de edad: 32 personajes $(43,2 \%)$ fueron adultos y $25(33,8 \%)$, jóvenes adultos, representando tres cuartas partes del total. En cuanto a la categoría de color de piel, encontramos que 50 personajes $(67,6 \%)$ fueron de color de piel blanco, 23 (31\%) fueron morenos, y un personaje $(1,4 \%)$ fue afrodescendiente.

La tabla 3 muestra la categoría de ocupaciones identificadas. La frecuencia más alta fue en servicios $(\mathrm{n}=20)$, seguido por periodismo/comunicaciones $(\mathrm{n}=8)$ y empresarios, deportistas y amas de casa $(\mathrm{n}=7)$ con la misma frecuencia. En 12 casos no se logró identificar la ocupación, pues los personajes aparecieron solo una vez (personajes de reparto), pero mantuvieron diálogo y pudieron analizarse.

En la tabla 4 se presenta la información referente a la orientación sexual.

En las categorías de características identitarias de género, roles, hipersexualización del personaje, relación entre personajes y ámbito de actuación, se realizaron pruebas de diferencias de medias para muestras independientes utilizando como factor el sexo de los personajes. Para realizar el análisis, primero se creó un índice para cada subcategoría, utilizando la media de presencia de la categoría en los episodios en los que aparecía el personaje.

En cuanto a las características identitarias de género, se reportan las subcategorías con diferencias significativas en la tabla 5. El resto de las subcategorías analizadas no tuvieron diferencias significativas por sexo. Puede observarse que los hombres mostraron más características relacionadas con ser exitoso, trabajador, propositivo y abnegado, mientras que las mujeres despliegan más características amorosas y tiernas, pero con una significancia menor $(\mathrm{p}<, 10)$. 


\begin{tabular}{ccccc} 
& Frecuencia & Porcentaje & $\begin{array}{c}\text { Porcentaje } \\
\text { válido }\end{array}$ & $\begin{array}{c}\text { Porcentaje } \\
\text { acumulado }\end{array}$ \\
\hline Niño & 1 & 1,35 & 1,35 & 1,4 \\
\hline Adolescente & 3 & 4,05 & 4,05 & 5,4 \\
\hline Joven adulto & 25 & 33,78 & 33,78 & 39,2 \\
\hline Adulto & 32 & 43,24 & 43,24 & 82,4 \\
\hline Adulto mayor & 13 & 17,57 & 17,57 & 100 \\
\hline Total & 74 & 100 & 100 & \\
\hline
\end{tabular}

Tabla 2. Grupos de edad de los personajes de la temporada 1 de Club de Cuervos ( $N=74$ )

Fuente: Elaboración propia.

\begin{tabular}{ccccc} 
& Frecuencia & Porcentaje & $\begin{array}{c}\text { Porcentaje } \\
\text { válido }\end{array}$ & $\begin{array}{c}\text { Porcentaje } \\
\text { acumulado }\end{array}$ \\
\hline Servicios & 20 & 27 & 27 & 43,2 \\
\hline Periodismo - comunicación & 8 & 10,8 & 10,8 & 81,1 \\
\hline Empresario & 7 & 9,5 & 9,5 & 59,5 \\
\hline Deportes & 7 & 9,5 & 9,5 & 70,3 \\
\hline Ama de casa & 7 & 9,5 & 9,5 & 91,9 \\
\hline Profesional & 5 & 6,8 & 6,8 & 50 \\
\hline Entrenador - director & 4 & 5,4 & 5,4 & 100 \\
\hline Servicios sexuales & 2 & 2,7 & 2,7 & 94,6 \\
\hline Trabajador del hogar & 1 & 1,4 & 1,4 & 60,8 \\
\hline Gobierno & 1 & 1,4 & 1,4 & 82,4 \\
\hline No especificado & 12 & 16,2 & 16,2 & 16,2 \\
\hline Total & 74 & 100 & 100 & \\
\hline
\end{tabular}

Tabla 3. Ocupación de los personajes de la temporada 1 de Club de Cuervos ( $\mathrm{N}=74$ )

Fuente: Elaboración propia.

\begin{tabular}{ccccc} 
& Frecuencia & Porcentaje & $\begin{array}{c}\text { Porcentaje } \\
\text { válido }\end{array}$ & $\begin{array}{c}\text { Porcentaje } \\
\text { acumulado }\end{array}$ \\
\hline Heterosexual & 28 & 37,8 & 37,8 & 37,8 \\
\hline Homosexual & 1 & 1,4 & 1,4 & 39,2 \\
\hline Pansexual & 1 & 1,4 & 1,4 & 40,6 \\
\hline Transgénero & 1 & 1,4 & 1,4 & 42 \\
\hline Sin orientación sexual clara & 43 & 58 & 58 & 100 \\
\hline Total & 74 & 100 & 100 & \\
\hline
\end{tabular}

Tabla 4. Orientación sexual de los personajes de la temporada 1 de Club de Cuervos ( $N=7$ )

Fuente: Elaboración propia. 


\begin{tabular}{ccc|cc|c|cc} 
& \multicolumn{2}{c}{ Hombre $\mathbf{( n = 5 3 )}$} & \multicolumn{2}{|c|}{ Mujer $\mathbf{( n = 2 1 )}$} & $\begin{array}{c}\text { Prueba de } \\
\text { Levene }\end{array}$ & \multicolumn{2}{c}{$\begin{array}{c}\text { Prueba T para la } \\
\text { igualdad de medias }\end{array}$} \\
\hline Media & D.T. & Media & D.T. & Sig. & $\mathbf{t}$ & Sig. \\
\hline Exitoso/a & $\mathbf{0 , 1 0}$ & $\mathbf{0 , 2 8}$ & 0,01 & 0,03 & $0,002^{* * *}$ & 2,38 & $0,021^{* *}$ \\
\hline Trabajador/a & $\mathbf{0 , 2 3}$ & $\mathbf{0 , 3 7}$ & 0,06 & 0,16 & $0,000^{* * *}$ & 2,67 & $0,009^{* * *}$ \\
\hline Amoroso/a & $\mathbf{0 , 0 2}$ & $\mathbf{0 , 0 7}$ & $\mathbf{0 , 0 6}$ & 0,16 & $0,000^{* * *}$ & $-2,07$ & $0,05^{*}$ \\
\hline Abnegado/a & $\mathbf{0 , 1 9}$ & $\mathbf{0 , 3 4}$ & 0,04 & 0,12 & $0,000^{* * *}$ & 2,66 & $0,01^{* *}$ \\
\hline Propositivo/a & $\mathbf{0 , 1 7}$ & $\mathbf{0 , 3 0}$ & 0,05 & 0,13 & $0,001^{* * *}$ & 2,37 & $0,02^{* *}$ \\
\hline Tierno/a & $\mathbf{0 , 0 5}$ & $\mathbf{0 , 1 3}$ & $\mathbf{0 , 1 7}$ & 0,27 & $0,000^{* * *}$ & $-1,99$ & $0,06^{*}$ \\
\hline
\end{tabular}

${ }^{* * *} \mathrm{p}<, 01 ;{ }^{* *} \mathrm{p}<, 05 ;{ }^{*} \mathrm{p}<, 10$

Tabla 5. Diferencia de medias entre hombres y mujeres en la expresión de características identitarias de género $(\mathrm{N}=74)$

Fuente: Elaboración propia.

En la categoría de roles, no se presentaron diferencias de sexo en toma de decisiones, proveedor/a, cuidador/a y parásito, pero sí en el rol de consejero/a ( $t$ $=-2,63, \mathrm{p}=, 010)$ : los hombres $(x=0,18$ D.T. $=0,31)$ reportaron una media superior a las mujeres $(x=0,05$ D.T. $=0,13)$.

En la hipersexualización de personajes no se encontraron diferencias significativas ( $t=-1,63, p=117)$, por lo que los hombres y mujeres que fueron incluidos en el análisis fueron hipersexualizados en la misma medida. Al respecto, es necesario considerar lo mencionado sobre los criterios de inclusión y exclusión, ya que una importante cantidad de mujeres que no tuvieron diálogos y que, por lo tanto, no fueron consideradas para el análisis, se presentaba de manera hipersexualizada.

En la relación entre personajes se obtuvieron diferencias significativas entre los grupos, tanto en la homosocialidad $(t=4,38 ; \mathrm{p}=, 000)$ como en la heterosocialidad $(\mathrm{t}=-2,66 ; \mathrm{p}=, 013)$; las mujeres tuvieron una media $(x=0,70 ; \mathrm{D} . \mathrm{T} .=0,58)$ superior a la de los hombres $(x=0,33$; D.T. $=0,43)$ en heterosocialidad y los hombres, una media superior $(x=0,99$; D.T. $=0,43)$ en homosocialidad $(x=0,45$; D.T. $=0,51)$.

En los ámbitos de actuación, no hubo diferencias significativas en lo público ni en lo doméstico, pero sí en lo privado $(t=-2,17 ; p=, 033)$, en donde la media de aparición de las mujeres $(x=0,46$; D.T. $=0,47)$ en ámbitos como el hogar fue superior a la de los hombres ( $x=0,23$ D.T. $=0,38)$. Es necesario reportar que, aunque aparecieron personajes realizando actividades domésticas (exclusivamente mujeres), en su mayoría fueron excluidas por no cumplir con el criterio de inclusión de mantener un diálogo. 
En cuanto a la reproducción de tópicos, se identificó que los personajes Hugo Sánchez (asistente personal de Chava Iglesias, protagonista de la serie), Tony (futbolista novato del equipo Cuervos), Juan Pablo (primo de los protagonistas, quien se dedica a labores de asistencia social) y Rafael (portero del equipo Cuervos y esposo de Isabel Iglesias, protagonista de la serie) son presentados con el tópico de chico/a bueno/a y, en algunos episodios, Isabel Iglesias (protagonista de la serie) y Moisés (jugador y capitán del equipo Cuervos) también reproducen el tema.

El rol de chico/a malo/a lo reproducen Chava Iglesias (presidente del equipo y protagonista de la serie), Aitor Cardoné (jugador internacional que se incorpora al equipo Cuervos por presión de Chava Iglesias), Eliseo Canales (agente de jugadores y antagonista de Chava e Isabel) y Potro (jugador internacional del equipo Cuervos) como personajes regulares, y otros no recurrentes como el señor Rubio (patrocinador del equipo Cuervos, quien acosa a Isabel) y Fernando (manager y pareja ocasional de Aitor Cardoné). La protagonista de la serie, Isabel Iglesias, se presenta en ocasiones como chica mala y como mujer masculinizada en la mayoría de los episodios de la temporada y es el único personaje que reproduce este tópico, mostrando formas de hablar con un tono hostil o agresivo, y también formas de vestir y de comportarse que le permiten sumergirse en un contexto primordialmente masculino.

El tópico de femme fatale principalmente lo reproducen Mary Luz (ama de casa y novia del padre de los protagonistas al momento de su muerte) y Paty Villa (periodista deportiva que se involucra sexualmente con Chava Iglesias), así como dos mujeres cuyos nombres no fueron mencionados, aunque tuvieron diálogo (una en el primer episodio y otra en el episodio 7).

El tópico de persona engañada lo reprodujeron en mayor medida personajes mujeres, tales como Gloria Iglesias (ama de casa y madre de Isabel Iglesias, quien fue engañada por Salvador Iglesias padre con la madre de Chava Iglesias), y Ximena (ama de casa y esposa de Moisés, quien se enteró del engaño por medios de comunicación). También fue reproducido por Isabel Iglesias, quien es engañada por su hermano cuando le roba un discurso que lo hace ganar la presidencia. El único personaje hombre que se representa como engañado es Chava Iglesias cuando es traicionado por su hermana y por Mary Luz.

Por último, 11 de los 13 episodios (84,62\%) no cumplieron con los criterios del test de Bechdel-Wallace; los dos en los que se cumplieron (15,38\%) fueron en una escena cada uno: en el episodio 3 , en el que en una conversación se aborda el tema del dinero (corrupción), y en el episodio 6, en donde el tema fue el embarazo. En el resto de los episodios, cuando hablan dos mujeres es sobre hombres, por lo que no se cumple el criterio o se cumple parcialmente. 


\section{DISCUSIÓN}

En la serie se identificó una subrepresentación de las mujeres y de miembros de las minorías sexuales, lo cual es concordante con lo encontrado en estudios previos (Menéndez \& Zurian, 2014). Por su parte, la sobrerrepresentación de personajes hombres en el área de deportes (específicamente fútbol) abona a la reproducción de estereotipos y roles construidos socialmente asociados con lo masculino que se han mostrado en otras series latinoamericanas que colocan como figuras centrales y protagónicas a los hombres, como se muestra en el estudio de Mateos-Pérez y Ochoa (2021). Además, resultó llamativo que la única mujer transgénero que aparece con diálogo (y otras mujeres transgéneros que no hablan) se muestra como laborante en el comercio sexual, lo cual podría estar sustentado y reforzar un prejuicio hacia los colectivos transexuales y transgénero. Asimismo, existe una sanción social constante hacia el personaje pansexual, que se manifiesta mediante burlas, críticas o prejuicios, y que incluso llegan a constituirse en discriminación.

Encuanto a la hipersexualización, se observó que no hay diferencias significativas por sexo; sin embargo, resulta interesante que los personajes hipersexualizados de ambos géneros están muy ceñidos a modelos de belleza occidental (delgadez, blanquitud, cuerpo atlético, rubios). Por otro lado, si bien no era un objetivo previsto en el estudio, resultó evidente que hay una subrepresentación de niños y adultos mayores; es decir, la serie está focalizada en un sujeto ideal joven o adulto joven. Asimismo, hay una minoría de personajes morenos.

En suma, es posible identificar un tipo de identidad masculina presentada constantemente en la temporada 1 de la serie Club de Cuervos que se caracteriza por ser hombre, heterosexual, blanco, adulto joven y de clase alta. Esto visibiliza los modelos de sujeto que prevalecen en culturas como la mexicana, en las que todavía permean idealizaciones y aspiraciones asociadas con lo eurocéntrico.

Con relación a las características identitarias de género, se encontró que se reproducen figuras y funciones estereotipadas, ya que a las mujeres se les presentó como amorosas y tiernas un mayor número de veces, mientras que a los hombres se les mostró como exitosos, trabajadores y propositivos, lo cual coincide con diversos estudios (Galán, 2007; Hidalgo-Marí, 2017; Fonseca, 2017; Menéndez \& Zurian, 2017). Cabe señalar que, en las características identitarias de amoroso y tierno, la diferencia -si bien fue significativa- fue pequeña, lo cual se puede deber al quiebre de las masculinidades hegemónicas y a la intención de presentar nuevos modelos de masculinidad. Así, en la serie se presentan algunos nuevos modelos de masculinidad representados por personajes protagónicos, secundarios protagónicos y no protagónicos, tales como Chava Iglesias (presidente del equipo de fútbol y uno de los protagonistas de la serie), Rafael (portero, entrenador y esposo 
de la protagonista), Hugo Sánchez (joven asistente personal del presidente del equipo de fútbol), y Tony (el más joven y novato de los jugadores de fútbol). Esta intención de mostrar nuevos modelos de masculinidad se ha documentado en otros estudios acerca de ficción televisiva (Menéndez \& Zurian, 2014; Mateos-Pérez \& Ochoa, 2021). En el caso de Mateos-Pérez y Ochoa (2021), en su estudio centrado en paternidades, aunque refieren que la identificación como padres forma parte de la narrativa de las series que analizaron (rompiendo con las masculinidades hegemónicas de ausencia), destaca que identificaron una paternidad patriarcal, lejana de afectividades y de acompañamiento, lo que no coincide con lo observado en la serie de nuestro estudio.

Algunas cualidades que se adjudican a estas nuevas masculinidades son la representación de hombres sensibles, comprensivos, consejeros, cuidadores, afectivos, pacientes y que hacen trabajo doméstico (tal como se puede apreciar en el personaje de Rafael), así como hombres que son detallistas, minuciosos, cuidadosos de su vestimenta e imagen física, sensibles y serviciales (tal como lo representa Hugo Sánchez). Incluso, hay figuras de hombres que se supeditan a las indicaciones de sus parejas mujeres (como lo encarna el personaje de Moisés, quien es futbolista y líder del equipo deportivo pero que reza cuando la esposa se lo indica o evita comentarios para que ella no se moleste).

También resultó llamativo que a los hombres se les presentó como más abnegados que a las mujeres. Estos hallazgos son contrarios a lo documentado por Menéndez y Zurian (2014), quienes sostienen que en las series de televisión y el cine a las mujeres se les representa más frecuentemente como abnegadas. Una posible explicación es que la gran mayoría de los personajes tienen como ocupación laboral los servicios (secretario, chofer, etc.). Estos servicios se ofrecen a los personajes principales, a quienes se presenta como empresarios y de clase alta, lo cual evidencia la intersección de categorías como género y clase. Otra posibilidad es que algunos de los personajes pertenecen a un equipo deportivo, donde hay jerarquías y normas que seguir para poder jugar. Cabe señalar que los personajes que se presentan como abnegados habitualmente son los más jóvenes, por ejemplo, jugadores recién incorporados al equipo deportivo. En futuros estudios acerca de ficción televisiva se podrían realizar análisis interseccionales para identificar la interconexión de variables como edad, orientaciones sexuales, género, clase social y raza, ya que identificamos que los estereotipos y roles están claramente diferenciados entre los sujetos de acuerdo con su posición social y circunstancias.

Con respecto a los roles no hubo diferencias significativas entre hombres y mujeres en la mayoría de los roles revisados. Tan solo se encontraron diferencias significativas en el rol de consejero, destacándose que los hombres son presentados 
más frecuentemente con esta característica. Si bien no era un objetivo central del estudio, se identificó que los hombres que ocupan el rol de consejeros son los más adultos, lo cual podría ser analizado de modo más puntual y focalizado en futuros estudios sobre ficción televisiva.

En la reproducción de tópicos se encontró que uno de los personajes protagónicos (Isabel) se muestra como una mujer masculinizada, quien destaca por su fuerza, capacidad para solución de conflictos, agresión, competitividad, habilidad para los negocios, raciocinio e inteligencia, lo cual es coincidente con lo que encontraron Hidalgo-Marí (2017) y Bonavitta y de Garay (2019) como un eje distintivo de las series en las que se muestra a las mujeres ocupando nuevos perfiles profesionales.

En categorías de roles, identidades y reproducción de tópicos, fue notable que muchas mujeres que sí tuvieron diálogos en la serie carecían de un nombre o de una descripción acerca de su profesión, intereses o actividades, por lo que solamente pudieron ser caracterizadas como esposas, madres, o amas de casa, lo que implica una reproducción de una identidad de género femenina hegemónica constituida por el ser para otros (Lagarde, 1990) y subordinada a los hombres (Bonavitta \& de Garay; 2019). En contraposición, los hombres en su mayoría tenían un nombre y al menos una ocupación.

En la categoría de relación entre personajes (homosociabilidad y heterosociabilidad), fue significativo que las mujeres socializaron más con los hombres que con otras mujeres. Además, en las ocasiones en las que conversaron con otras mujeres, muchas veces terminaban hablando de hombres. Por ello, no se pasó el test de Bechdel-Wallace en 11 de los 13 episodios de la temporada. Por el contrario, los hombres tenían más diálogos con otros hombres. Asimismo, estos temas no estaban centrados en las mujeres, sino que versaban en torno al trabajo y los proyectos personales. Cuando los hombres interactuaban con mujeres, en general podía observarse una objetualización de las mismas, lo cual es coincidente con otros estudios (Bird, 1996). Es de destacar que las diferencias en la socialización pudieron ser causadas por el número de personajes masculinos y femeninos, considerablemente mayor en el caso de los hombres, lo en sí fue un dato significativo para ver la subrepresentación de las mujeres en la serie.

Por último, fue relevante notar que sí se presenta más veces a las mujeres que a los hombres en el espacio privado. Sin embargo, las y los personajes que se muestran en este espacio no están haciendo tareas domésticas; es decir, lo privado se construye como un espacio lúdico, de descanso, de deporte, de continuidad del trabajo o de despliegue de la sexualidad, lo cual podría deberse a que la serie retrata y se focaliza en la vida de personas de clase alta que habitualmente tienen choferes, asesoras del hogar y personal que realiza el trabajo doméstico. 
Tan solo dos personajes que tenían diálogo en la serie se presentaron en el espacio privado realizando actividades domésticas, es decir, en lo que en este trabajo se denominó espacio doméstico. Estos personajes fueron una empleada doméstica y Rafael. Conviene remarcar que Rafael es un personaje que a lo largo de la serie se presenta con una masculinidad deconstruida, que muestra rasgos y características asociadas tradicionalmente con lo femenino.

Un aspecto a destacar visibilizado en la serie fue el tema de la raza y el nivel socioeconómico que, aunque excedía los límites del objetivo de este estudio, fueron evidentes, ya que hay una sobrerrepresentación de personajes blancos comparados con resultados de la Encuesta Nacional de Discriminación (Consejo Nacional para Prevenir la Discriminación [CONAPRED], 2017)², y estos ocupan puestos de poder o liderazgo, además de una focalización en los estilos de vida de las clases altas. Por ello, se considera que será importante recuperar y analizar con detalle estos temas en futuras investigaciones acerca de ficción televisiva en México.

Una limitante para considerar en nuestro estudio fue que eliminamos a diversos personajes mujeres que pudieran dar pie a análisis en términos dehipersexualización y labores de cuidado del hogar que consideramos como categorías de análisis. Sin embargo, esto es un dato relevante que exhibe la objetualización de las mujeres en esta serie de ficción televisiva.

Esto en conjunto muestra que, aún en una serie moderna, en donde se rompe con muchos tabúes en torno a la presentación de orientaciones sexuales o en la hipersexualización, se siguen reproduciendo a nivel de personajes los mismos estándares, roles y estereotipos que en la mayoría de las series, lo que coincide con lo reportado por otras autoras (Bonavitta \& de Garay, 2019). No obstante, es relevante destacar que también se identificaron transiciones, puesto que contrario a lo que proponen algunos autores (Bonavitta \& de Garay, 2019; Galán, 2007; Sánchez et al. citado en Hidalgo-Marí, 2017; Hidalgo-Marí, 2017; Menéndez \& Zurian, 2014), en esta serie no se identificó que en las mujeres se resalte el rol de madre, ni las nociones de amor romántico o el ideal de pareja tradicional, aunque sí se sigue presentando con mayor frecuencia a las mujeres como engañadas. Finalmente, es relevante destacar que, si bien han cambiado las plataformas y los modos de producir cine y ficción televisiva, los contenidos siguen reflejando y reproduciendo desigualdades de género.

1. Mientras que la Encuesta Nacional de Discriminación 2017 se refiere que 29,4\% de las personas se considera blancas, en nuestro estudio encontramos $67,6 \%$. 


\section{REFERENCIAS}

Baron, R. \& Byrne, D. (2005). Psicología Social (Social Psychology). Madrid: Pearson educación.

Berger, P. \& Luckmann, T. (2001). La construcción social de la realidad (The Social Construction of Reality). Amorrortu Editores.

Bird, S. (1996). Welcome to the men's club: homosociality and the maintenance of hegemonic masculinity. Gender \& Society, 10(2), 120-132. https://doi.org/10.1177/089124396010002002

Bueno-Fischer, R. (2006). El ejercicio de ver: medios y educación (The exercise of seeing: media and education). In I. Dussel \& D. Gutiérrez (Comps.), Educar la mirada. Políticas y pedagogías de la imagen (Educate the gaze. Policies and pedagogies of the image) (pp. 165-177). Manantial/Flacso Argentina/Fundación Gosde.

Bonavitta, P. \& de Garay, J. (2019). La casa de papel, Rita y Merlí: entre nuevas narrativas y viejos patriarcados (La casa de papel, Rita and Merlí: between new narrativ0es and old patriarchates). Investigaciones Feministas, 10(2), 207-221. https://doi.org/10.5209/infe.66490

Chiver, M., Zimbron, L., \& Vargas, M. (Producers). (2015-2019). Club de Cuervos (TV series). Netflix.

Caballero-Gálvez, A. \& Herrero-Jiménez, B. (2017). Representaciones de género en las redes móviles de contactos. Cuerpo e identidad en "Adopta un tío" (Gender representation on mobile dating apps. Body and identity in "Adopta un tío"). Prisma Social, (2), 31-56. https://revistaprismasocial.es/article/view/1549/1749

Castañeda, L. (2016). La configuración de género en mujeres profesionistas no madres en Guadalajara, México (The configuration of gender identify in professional women who are not mothers in Guadalajara, Mexico) (Unpublished doctoral dissertation). Ciesas Occidente.

Consejo Nacional para Prevenir la Discriminación (CONAPRED). (2017). Encuesta Nacional sobre Discriminación 2017 (2017 Discrimination National Survey). Comisión Nacional de Derechos Humanos de México, Conacyt, Inegi. https://www.conapred.org.mx/userfiles/files/PtcionENADIS2017_08.pdf

Fonseca, J. A. (2017). Representaciones narrativas de la mujer en el cine de ficción costarricense (Narrative representations of women in Costa Rican fiction cinema) (2008-2012). Cuadernos Intercambio sobre Centroamérica y el Caribe, 14(2), 77-95. https://revistas.ucr.ac.cr/index.php/intercambio/article/view/30947

Galán, E. (2006). Personajes, estereotipos y representaciones sociales. Una propuesta de estudio y análisis dela ficción televisiva (Characters, stereotypes and social representations. A proposal for the study and analysis of television fiction).ECO-POS, 9(1), 58-81.http://hdl.handle.net/10016/9475

Galán, E. (2007). Construcción de género y ficción televisiva en España (Gender construction and fiction TV). Comunicar. Revista Científica de Comunicación y Educación, 28(14), 229-236. https://www.revistacomunicar.com/ojs/index.php/comunicar/article/view/C28-2007-28

Goffman, E. ([1959] 2001). La presentación de la persona en la vida cotidiana (The Presentation of Self in Everyday Life). Amorrortu Editores. 
Guarinos, V., Gordillo, I., Ramírez-Alvarado M., Jiménez, J., \& Hermida, A. (2019). Masculinidades de ficción televisiva y retroalimentación online. Jóvenes y adolescentes enredados fuera y dentro de Física o química y El Internado (Television fiction masculinities and online feedback. Young people and teenagers entangled inside and outside of Physics or Chemistry and The Internship). In Comunicación y desarrollo en la era digital. II Congreso Internacional de la AE-IC. Asociación Española de Investigadores de la Comunicación. http://hdl.handle.net/11441/25463

Hayes, A. F. \& Krippendorff, K. (2007). Answering the call for a standard reliability measure for coding data. Communication Methods and Measures, 1(1), 77-89.

https://doi.org/10.1080/19312450709336664

Hidalgo-Marí, T. (2017). De la maternidad al empoderamiento: una panorámica sobre la representación de la mujer en la ficción española (From motherhood to empowerment: an overview of the representation of women in Spanish TV fiction). Prisma Social, (2), 291-314. https://revistaprismasocial.es/article/view/1551

Krippendorff, K. (2004). Content analysis: An introduction to its methodology. Sage.

Park, B., Judd, C. M., \& Ryan, C. S. (1991). Social categorization and the representation of variability information. In W. Stroebe \& M. Hewstone (Eds.), European Review of Social Psychology, vol. 2 (pp. 211-245). Wiley.

Lagarde, M. (1990). Identidad femenina (Feminine identity). CIDHAL.

Mateos-Pérez, J. \& Ochoa, G. (2016). Contenido y representación de género en tres series de televisión chilenas de ficción (2008-2014) (Chilean televisions series. Themes, content and gender representation (2008-2014)). Cuadernos.info, (39), 55-66. https://doi.org/10.7764/cdi.39.832

Mateos-Pérez, J. \& Ochoa, G. (2021). La representación de la paternidad en series de televisión chilenas del siglo XXI (The representation of fatherhood in Chilean television series of the XXI century). Cuadernos del Centro de Estudios de Diseño y Comunicación, 22(95), 51-64. https://doi.org/10.18682/cdc.vi95.3908

Menéndez, M. \& Zurian, F. (2014). Mujeres y hombres en la ficción televisiva norteamericana hoy (Women and Men in the American Television Fiction Today). Anagramas Rumbos $y$ Sentidos de la Comunicación, 13(25), 55-71. https://doi.org/10.22395/angr.v13n25a3

Nájar, A. (2015, August 7). Cómo será “Club de Cuervos”, la primera serie de Netflix en español (All about Club de Cuervos, Netflix’s first Spanish-speaking series). BBC Mundo. https://www.bbc.com/mundo/noticias/2015/08/150806_club_cuervos_primera_serie_ espanol_netflix_an

Netflix. (n.d.). Club de Cuervos. https://www.netflix.como/mx/title/800030346

Freitas, J., Rosenzvit, M., \& Muller, S. (2016). Automatización del Test de Bechdel-Wallace (Automation of the Bechdel-Wallace Test). Ética y Cine Journal, 6 (3), 35-40. http://doi.org/10.31056/2250.5415.v6.n3.16498

Ortega, M. \& Simelio, N. (2010). La representación de mujeres trabajadoras en series de máxima audiencia (The representation of women workers in the series of maximum audience broadcast in Spain (2010)). Comunicación, 10 (1), 1006-1016. Retrieved from https://idus.us.es/handle/11441/36466 
Ortiz, G. (2020, March 9). Internet vs. televisión: la contienda por la audiencia (Internet vs. Television: fighting for audience). El Economista. https://www.eleconomista.com.mx/ empresas/Internet-vs.-television-la-contienda-por-la-audiencia-20200309-0084.html

Palomar, C. (2007). Maternidad en prisión (Motherhood in prison). Universidad de Guadalajara.

Pérez, J. (2017). Mujeres en el videoclip: la representación de la mujer en vídeos musicales de Diane Martel (Women in the music video: Representation of the woman in the music videos by Diane Martel). Prisma Social, (2), 202-232. https://revistaprismasocial.es/article/view/1550

Ramírez, J. (2019). "Me da mucho miedo esto": Hombres, (des)empleo y familia. Un acercamiento al vocabulario emocional ("I'm very afraid of this": Men, (un)employment and family. An approach to emotional vocabulary). Revista Interdisciplinaria de Estudios de Género de El Colegio de México, 5, 1-34. https://doi.org/10.24201/reg.v5i0.402

Richard, N. (2006). Estudios visuales y políticas de la mirada (Visual and political studies of the gaze). In I. Dussel \& D. Gutiérrez, (Comps.). Educar la mirada. Políticas y pedagogías de la imagen (Educate the gaze. Policies and pedagogies of the image) (pp. 97-109). Manantial/ Flacso Argentina/Fundación Gosde.

Scott, J. (1999). El género: una categoría útil para el análisis histórico (Gender: a useful category for historical analysis). In M. Lamas (Comp.), El género: la construcción cultural de la diferencia sexual (Gender: the cultural construction of sexual difference) (pp. 265-302). PUEG.

Scott, J. (2008). Género e historia (Gender and history). Universidad Autónoma de México.

Simelio, N. (2010). La Representación de las Relaciones Sociales en las series de ficción digitales creadas específicamente para internet. La televisión como contribución a la alfabetización digital (The representation of social relationships in digital fiction series created specifically for the Internet. Television as a contribution to digital literacy). In Proceedings of the Congreso Euro-Iberoamericano de Alfabetización Mediática y Culturas Digitales (Euro-Ibero-American Congress on Media Literacy and Digital Cultures) (pp. 1-11). http://hdl.handle.net/11441/56278

Siri, L. (2016). El rol de Netflix en el ecosistema de medios y telecomunicaciones ¿el fin de la televisión y el cine? (Netflix's role in the media and telecommunications ecosystem: the end of television and cinema?) Hipertextos, 4(5), 47-109. http://revistahipertextos. org/wp-content/uploads/2016/11/El-rol-de-Netflix-en-el-ecosistema-de-medios-ytelecomunicaciones.-Siri.pdf

Suárez-Cousillas, T., Martínez-Fernández, V. A., \& Sánchez-Amgoage, E. (2019). Audiencia de plataformas SVOD. El caso de Netflix, Blockbuster, Hulu y HBO (SVOD Platform Audience. The Case of Netflix, Blockbuster, Hulu and HBO). In Proceedings from the 14th Iberian Conference on Information Systems and Technologies, 1-6. https://doi.org/10.23919/CISTI.2019.8760790

Souza, M. (2009). La artesanía de la investigación cualitativa (The art of qualitative research). Lugar Editorial. 


\section{SOBRE LAS AUTORAS}

KARLA CONTRERAS, Doctora en Ciencias Sociales con especialidad en Antropología Social en CIESAS Occidente (México). Maestra en Psicología Social por la Universidad Católica del Norte (Chile). Licenciada en Psicología por el Centro Universitario de la Ciénega, Universidad de Guadalajara. Profesora e Investigadora de Tiempo Completo del Departamento de Economía y Ciencias Políticas del Centro Universitario de Tonalá, Universidad de Guadalajara. México. Miembro del GT Clacso Luchas antipatriarcales, familias, géneros, diversidades y ciudadanía. Líneas de investigación: género y subjetividades.

iD https://orcid.org/0000-0001-6061-4600.

LAURA ALFARO, Doctora en Psicología con Orientación en Calidad de Vida y Salud por la Universidad de Guadalajara. Maestra en Ciencias Forenses y Criminología con orientación en Criminología. Licenciada en Psicología. Profesora de Tiempo Completo del Departamento de Comunicación y Psicología del Centro Universitario de la Ciénega de la Universidad de Guadalajara. Líneas de investigación: Factores psicosociales, emociones y construcción del bienestar; Universidad, juventud y bienestar; Poder, intersubjetividad y vida cotidiana.

iD https://orcid.org/0000-0002-9857-7339. 